\title{
Influence of Polypyrrole Incorporated Electrospun Poly (vinylidene fluoride-co-hexafluoropropylene) Nanofibrous Composite Membrane Electrolyte on the Photovoltaic Performance of Dye Sensitized Solar Cell
}

\author{
Vijayakumar Elayappan, ${ }^{1,2}$ Vignesh Murugadoss, ${ }^{1}$ Zhaofu Fei, ${ }^{3}$ Paul J. Dyson, ${ }^{3}$ and Subramania Angaiah ${ }^{1, *}$
}

\begin{abstract}
Electrospun poly(vinylidene fluoride-co-hexafluoropropylene) (PVdF-HFP) nanofibrous composite membranes (esCPMs) incorporated with various wt\% of polypyrrole (PPy) nanoparticles (1, 2, 3 and 4 wt\%) were prepared by electrospinning technique for dye sensitized solar cell (DSSC) applications. The surface morphology, crystallinity, porosity and electrolyte uptake of the prepared esCPMs were examined. The esCPMs were soaked in an ionic liquid electrolyte containing $0.5 \mathrm{M}$ Lil, $0.05 \mathrm{M} \mathrm{I}_{2}, 0.5 \mathrm{M} \mathrm{4-tert} \mathrm{butyl} \mathrm{pyridine} \mathrm{and} 0.5 \mathrm{M}$ 1-Butyl-3-methylimidazolium iodide in acetonitrile to get electrospun PVdF-HFP/PPy nanofibrous composite polymer membrane electrolytes (esCPMEs). The influences of PPy on ionic conductivity and electrocatalytic activity of esCPMEs were studied by electrochemical impedance and Tafel polarization measurements. The DSSC fabricated with esCPME ( $2 \mathrm{wt} \%$ PPy) had the photo-conversion efficiency (PCE) of $7.02 \%$ at $100 \mathrm{~mW}$ $\mathrm{cm}^{-2}$, which was higher than that fabricated with the conventional electrospun PVdF-HFP membrane electrolyte (esPME).
\end{abstract}

Keywords: Electrospinning; Polymer membrane; Nanocomposite; Polymer electrolyte; Dye-sensitized solar cell.

Received date: 3 March 2020; Accept date: 2 May 2020

Article type: Research article

\section{Introduction}

Dye-sensitized solar cells (DSSCs) are a remarkable renewable energy source and an attractive alternative for conventional $\mathrm{p}$-n junction solar cells due to their high efficiency, low cost, ease of fabrication and transparency. ${ }^{[1]}$ Their photoconversion efficiency (PCE) has grown from 7\% to $14 \%{ }^{[2]}$ Despite numerous attractive properties of DSSCs, their long-term operation remains a challenge due to the evaporation and leakage of the electrolyte. ${ }^{[3]}$ Efforts have been taken to improve the long-term stability of DSSCs by replacing liquid electrolytes with non-volatile ionic liquids and quasi-solid state electrolytes. ${ }^{[4-6]}$ Among various kinds

\footnotetext{
${ }^{1}$ Electro-Materials Research Laboratory, Centre for Nanoscience and Technology, Pondicherry University, Puducherry - 605 014, India.

2 Department of Materials Science and Engineering, Korea University, Seoul-02841, Republic of Korea.

${ }^{3}$ Institut des Sciences et Ingenierie Chimiques, Ecole Polytechnique Federale de Lausanne (EPFL) CH-1015, Lausanne, Switzerland

*Corresponding Author

E-mail: a.subramania@gmail.com
}

of quasi-solid-state electrolytes, gel polymer electrolyte has attracted great attention due to their non-flammability, negligible vapor pressure, conductivity, excellent long term stability, etc. ${ }^{[7-9]}$ However, DSSCs assembled using polymer gel electrolyte tend to have a low PCE, when compared to the same device assembled by using liquid electrolytes. The lower PCE is mainly due to the low ion conductivity of polymer gel electrolytes leading to a large resistance in transferring the charges. The high porosity and large surface area polymers with the capacity of absorbing large quantity of ionic liquid electrolyte within the microporous interconnected pores, allowing free movement, can overcome this problem. ${ }^{[10-13]}$ Moreover, conducting polymers integrated gel electrolytes have been reported. ${ }^{[14,15]}$ Polymer gel electrolyte incorporated with conducting polymers increases the electrical conduction of the polymer electrolyte. These conducting channels reflux the electrons from the counter electrode. This enhances the electrocatalytic reaction of triiodide to iodide from the counter electrode/polymer gel electrolyte interface, which increases the light-to-electricity conversion. ${ }^{[16]}$ 
Recently, conducting polypyrrole (PPy) incorporated three-dimensional poly(hydroxyethyl methacrylate/glycerol) gel electrolyte has been developed and increases the ionic conductivity, transport kinetics and electrocatalytic activity of iodide and triiodide redox couple that yield a reasonable PCE. ${ }^{[17]}$ The PPy also incorporated in a microporous poly(acrylic acid)-poly(ethylene glycol) matrix, which has increased the ionic conductivity and electrocatalytic activity of iodide in DSSC and achieved a reasonable PCE. ${ }^{[18]}$

Electrospun membrane has specific properties such as high surface/volume and aspect ratios, leading to low density and high pore volume and mechanical strength. ${ }^{[19,20]}$ The three-dimensional interconnected framework entraps the absorbed ionic liquid electrolyte. The movement of imbibed ionic liquid electrolyte in the electrospun polymer membrane is similar to that of liquid electrolyte. Thus, the electrospun polymer membrane electrolyte (esPME) offers the advantage of gel polymer electrolyte as well as the liquid electrolyte. $^{[21]}$ In addition, the integration of nanofiller reduces the crystallinity of polymer and facilitates the redox couple transportation and thereby enhances the ionic conductivity of esCPME. ${ }^{[22,23]}$ In our previous work, we have successfully developed the polyaniline incorporated electrospun PVdF-HFP membrane electrolyte to improve the performance of DSSC. ${ }^{[24]}$ To the best of our knowledge, PPy incorporated electrospun poly(vinylidene fluoride-cohexafluoropropylene) (PVdF-HFP) membrane electrolyte for DSSC applications has not been studied. Hence, in the present investigation, PPy incorporated esCPMEs were developed and their photovoltaic performance of DSSC was studied in details and compared with the conventional esPME.

\section{Experimental}

\subsection{Materials}

Polypyrrole nanoparticles were prepared by using a literature protocol. ${ }^{[15]}$ Acetone, and N, N'-dimethylacetamide were obtained from Merck, India Ltd. Lithium iodide, iodine, 4-tert-butyl pyridine, acetonitrile, and Di-tetrabutylammonium cis-bis (isothiocyanato) bis (2, 2'-bipyridyl-4, 4'-dicarboxylato) ruthenium (II) (N719 dye) were obtained from SigmaAldrich and PVdF-HFP from Arkema (Kynar flex 2801). Standard $\mathrm{TiO}_{2}$ paste and platinum paste were purchased from Dyesol Ltd. All these chemicals were analytical grade and used as received without any further purification.

\subsection{Preparation of esCPMEs}

The esCPMs were prepared by electrospinning technique by using $16 \mathrm{wt} \%$ solution of PVdF-HFP containing different wt $\%$ (i.e., 1, 2, 3 and $4 \mathrm{wt} \%$ ) of PPy in a mixture of acetone/N, N'-dimethyl acetamide $\left(7: 3\right.$ wt.\%). ${ }^{[25]}$ The mixture was mechanically stirred for $12 \mathrm{~h}$ and then loaded into a syringe. This solution was supplied to a stainless-steel needle (27 G) maintained at a high voltage of $19 \mathrm{kV}$ using a syringe pump at a flow rate of $0.5 \mathrm{~mL} \mathrm{~h}^{-1}$. The esCPM mats were collected from the collector plate, which was maintained at a distance of $12 \mathrm{~cm}$ from the needle tip. The electrospinning process was carried out at $25{ }^{\circ} \mathrm{C}$ and a relative humidity $(\mathrm{RH})$ of $35 \%$. The resulting esCPMs were removed and vacuum-dried at $80{ }^{\circ} \mathrm{C}$ for $12 \mathrm{~h}$ to remove the residual solvent. Similarly, the electrospun PVdF-HFP membrane (esPM) was prepared by adopting the above-said procedures without incorporation of PPy nanoparticles. The thicknesses of esCPMs was reduced from ca. 30 to $15 \mu \mathrm{m}$ by hot pressing. ${ }^{[26]}$ They were soaked in an ionic liquid electrolyte containing $0.5 \mathrm{M}$ LiI, $0.05 \mathrm{M} \mathrm{I}_{2}, 0.5 \mathrm{M}$ 4-tert butylpyridine and $0.5 \mathrm{M}$ 1-Butyl-3-methylimidazolium iodide in acetonitrile to obtain their corresponding esCPMEs.

\subsection{Characterization of esCPMEs}

The surface morphologies of esPM and esCPMs were studied by using a field emission scanning electron microscopy (JSM$7600 \mathrm{~F}$ ). The crystallinity of esPM and different $\mathrm{wt} \%$ of PPy incorporated esCPM was examined by X-Ray diffractometer (Rigaku, Ultima IV) using $\mathrm{Cu} \mathrm{K}_{\alpha}$ radiation with a step scanning of $0.05^{\circ}$ over the range of $20-80^{\circ}$. Differential scanning calorimetry (DSC) was performed at a heating rate of $10^{\circ} \mathrm{C} / \mathrm{min}$ under a nitrogen atmosphere in the temperature range between 30 and $200^{\circ} \mathrm{C}$ (TA Instruments; Q600 SDT). The heat history effects were removed by cooling the samples from $200{ }^{\circ} \mathrm{C}$ at $5{ }^{\circ} \mathrm{C} / \mathrm{min}$.

The crystallinity $\left(X_{c}\right)$ of the esCPMs was calculated by using Equation (1): $:^{[27]}$

$$
X_{c}(\%)=\frac{\Delta H_{m}^{\text {sample }}}{\Delta H_{m}} \times 100
$$

where $\Delta H_{m}^{\text {sample }}$ is the heat of melting for the sample, $\Delta H_{m}$ is the heat of melting for the pure PVdF, $104.7 \mathrm{~J} / \mathrm{g}$.

The porosity $(P)$ of esCPMs was determined by using Equation (2):

$$
P=\frac{m_{a} / \rho_{a}}{m_{a} / \rho_{a}+m_{p} / \rho_{p}}
$$

where $m_{a}$ is the weight of esCPM after impregnation with 1-butanol, $m_{p}$ is the weight of esCPM before impregnation with 1-butanol, and $\rho_{a}$ and $\rho_{b}$ are the density of 1-butanol and the dried esCPM, respectively.

To determine the electrolyte uptake of esCPM, the membrane was soaked in the appropriate electrolyte for $24 \mathrm{~h}$ and then removed from the electrolyte solution and excess electrolyte was removed by wiping. Electrolyte uptake was estimated by using Equation (3):

$$
U(\%)=\left[\left(m-m_{o}\right) / m_{o}\right] \times 100
$$

where $m$ and $m_{o}$ are the mass of esCPMs and esCPMEs, respectively.

The leakage of the electrolyte was calculated by using 
Equaiton (4): ${ }^{[28]}$

$$
R=\frac{M_{P E}}{M_{P E, \text { saturated }}}
$$

where $R$ is the relative absorption ratio of the liquid electrolyte, $M_{P E \text {,saturated }}$ is the mass of esCPMEs when the membrane is fully saturated with the liquid electrolyte, and $M_{P E}$ is the mass of the esCPME after a time interval when the saturated polymer membrane electrolyte is pressed between a filter paper.

The esCPMs were soaked in the liquid electrolyte and the ionic conductivity of resulting esCPMEs was measured by sandwiching the esCPME in between two stainless steel block electrodes using an electrochemical impedance technique at an $\mathrm{AC}$ amplitude of $10 \mathrm{mV}$ over the frequency range of $100 \mathrm{kHz}$ to $1 \mathrm{mHz}$ at the open circuit potential (Electrochemical analyzer, Biologic-VSP, France). The ionic conductivity $(\sigma)$ of esCPME was calculated using Equation (5):

$$
\sigma=\ell / R A
$$

where $\ell$ is the esCPME thickness, $A$ is the area of the esCPME and $R$ is the bulk resistance. The Tafel polarization measurements were carried out by using a symmetric cell consisting of $\mathrm{FTO} / \mathrm{Pt} / \mathrm{PMEs} / \mathrm{Pt} / \mathrm{FTO}$ to confirm the electrocatalytic activity of esCPMEs and esPME.

\subsection{DSSC fabrication}

The detailed procedure for the fabrication of DSSCs has been described previously. ${ }^{[29]}$ Briefly, titania paste was spread over the spacer between the scotch tape on the conducting glass substrate using the doctor blade technique and then sintered in air at $450{ }^{\circ} \mathrm{C}$ for $30 \mathrm{~min}$. After cooling to $80{ }^{\circ} \mathrm{C}$, the $\mathrm{TiO}_{2}$ electrodes were immersed in $0.3 \mathrm{mM}$ $\mathrm{N} 719$ dye solution for $12 \mathrm{~h}$ at $30{ }^{\circ} \mathrm{C}$. The DSSC was assembled using the esCPM by sandwiching a slice between the dye-sensitized $\mathrm{TiO}_{2}$ photoanode and $\mathrm{Pt}$ counter electrode using $60 \mu \mathrm{m}$ thick hot melt thermoplastic sealer (Surlyn). The ionic liquid electrolyte solution containing $0.5 \mathrm{M} \mathrm{LiI}$, $0.05 \mathrm{M} \mathrm{I}_{2}, 0.5 \mathrm{M}$ 4-tert butyl pyridine and $0.5 \mathrm{M}$ 1-Butyl3-methylimidazolium iodide in acetonitrile was injected into sealed electrode through one of the two small holes pre-drilled in the counter electrode. Similarly, the DSSC based on esPME was also assembled for comparison. ${ }^{[24]}$ The performance of the DSSCs was determined by using a calibrated AM 1.5 solar simulator (Newport, Oriel Instruments, Model: 67005) with a light intensity of 100 $\mathrm{mW} \mathrm{cm} \mathrm{cm}^{-2}$ and a computer-controlled digital source meter (Keithley, Model: 2420). Data for the DSSCs were accumulated over at least three measurements carried out under the same condition.

The long-term stability test was also carried out for DSSCs assembled with esCPME and esPME by recording their J-V curves every $48 \mathrm{~h}$ over a period of 30 days at an illumination light intensity of $100 \mathrm{~mW} \mathrm{~cm}$.

\section{Results and Discussion}

\subsection{Characterization of membranes}

The FE-SEM image of esPM and esCPM (2wt.\% PPy) reveals a three-dimensional network structure (Fig. 1), with fully interconnected pores and multi-fibrous layers, having fiber diameter of typically $300-400 \mathrm{~nm}$. It is observed that esCPM (2wt.\% PPy) have spherical structure in the nanofibers. This is resulted from increased net charge density of the PVdF-HFP solution after adding PPy that exert a force in the jet to form PPy spherical structures in the nanofibers. ${ }^{[24]}$ The pores appear to be fully interconnected and have a narrow pore size distribution capable of holding a large amount of liquid electrolyte. ${ }^{[30]}$ Indeed, the fibers swell on soaking in the ionic liquid electrolyte (ILE) and show a high electrolyte uptake. The ionic conductivity largely depends on the pore structure that entraps large amount of electrolyte and hence the formation of pores in the $3 \mathrm{D}$ network is very important to obtain a proper channel for ionic conduction. The electrolyte uptake of esCPM rapidly increased with increasing the soaking time in few minutes and then slowed down before reaching an equilibrium state. The esCPM with $2 \mathrm{wt} \%$ of incorporated PPy (esCPM (2wt.\% PPy)) exhibited a maximum porosity of $90.50 \%$ as determined by n-butanol method. This membrane absorbed $383 \%$ of electrolyte within a few hours, the uptake rate being faster than that of porous membranes obtained by other methods. The esCPME retained a large proportion of the absorbed electrolyte and had a very low leakage, ca. $0.2 \%$.
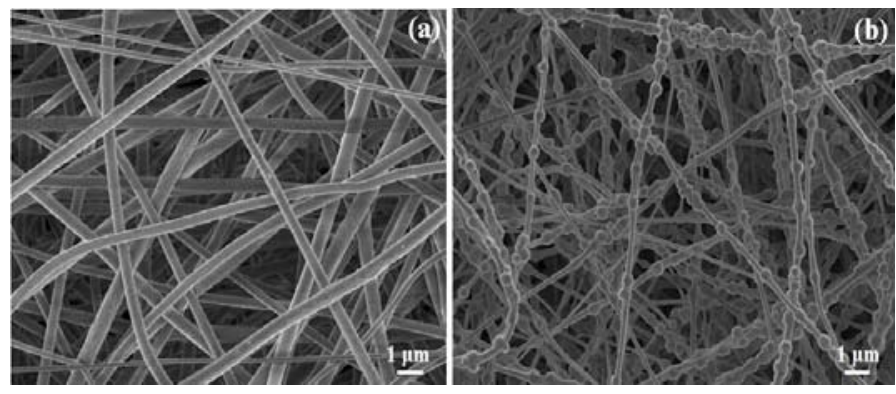

Fig. 1 FE-SEM image of (a) esPM and (b) esCPM(2wt\% PPy).

\subsection{XRD studies}

The variation in the degree of crystallinity of esCPMs was assessed by X-ray diffraction analysis (Fig. 2). A broad peak in the region of $15^{\circ}<2 \theta<30^{\circ}$ revealed that the PPy is amorphous in nature. The characteristic peaks corresponding to (100) and (020) planes at $18.2^{\circ}$ and $20^{\circ}$, respectively confirm the semi-crystalline nature of PVdF-HFP polymer. The intensity corresponding to these peaks is reduced considerably with increasing the concentration of PPy, showing that the additive reduces the crystallinity of the system. ${ }^{[31]}$ The lowest crystallinity is found at $2 \mathrm{wt} \%$ of PPy incorporated esCPM. When a higher content of PPy (beyond $2 \mathrm{wt} \%$ ) is added, the increased crystallization of PVdF-HFP is attributed to the agglomeration of PPy nanoparticles. The 
increased amorphocity could facilitate the migration of ions in the electrolyte that enhanced the ionic conductivity.

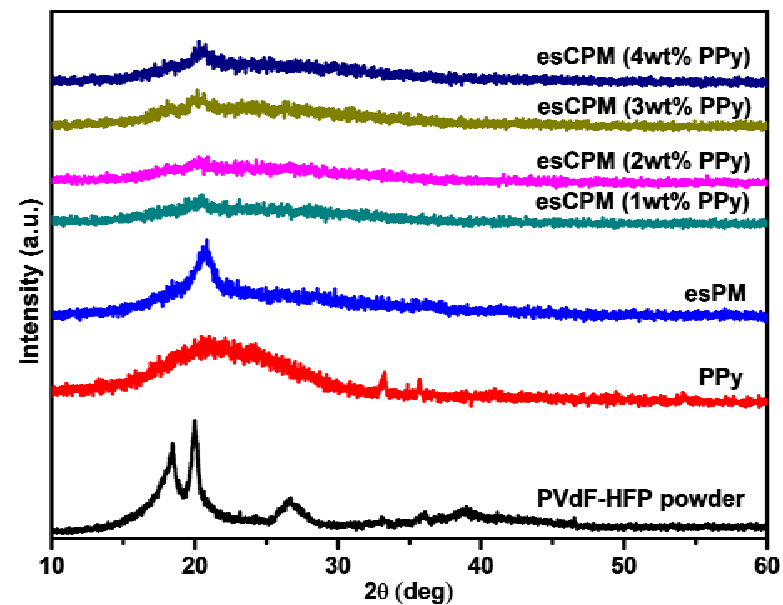

Fig. 2 XRD patterns of PVdF-HFP powder, PPy, esPM and different $w t \%$ of PPy incorporated esCPMs.

\subsection{DSC studies}

DSC thermograms of esPM and $2 \mathrm{wt} \%$ PPy incorporated esCPM contain an endothermic peak at 165 and $167{ }^{\circ} \mathrm{C}$, respectively (Fig. 3). The DSC curve obtained from the PVdF-HFP membrane shows only the removal of absorbed moisture and the melting of the polymer. The observed enthalpy values of the endothermic peaks due to melting are used to calculate the percentage of crystallinity using Equation 1, which corresponds to 31.96 and $30.23 \%$ for esPM and esCPM, respectively. This result is well consistent with the XRD data. Additionally, the difference in crystallinity indicates that the addition of PPy hinders the crystallization of PVDF-HFP chains. The reduced crystallinity provides a favorable conduction path for the charge carriers that leads to an increase in the ionic conductivity of the resulting electrolyte system. ${ }^{[32]}$

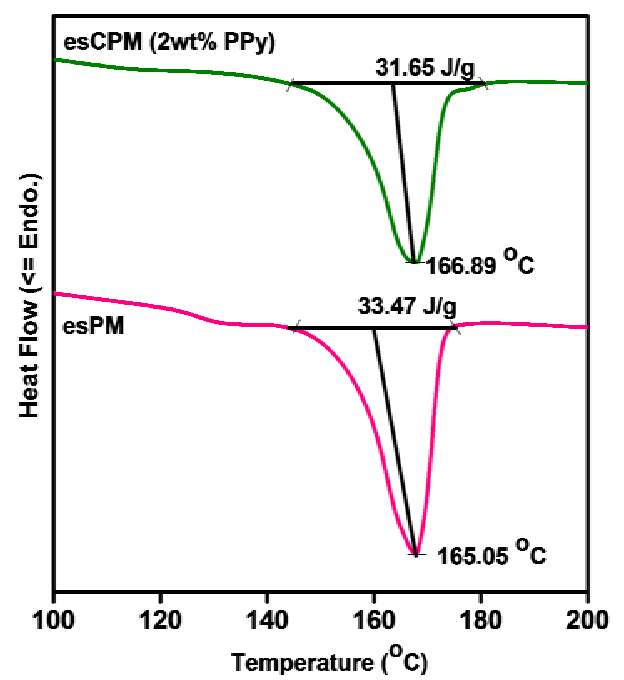

Fig. 3 DSC analysis of esPM and $2 \mathrm{wt} \%$ PPy-esCPM.

\subsection{Ionic conductivity studies}

Ionic conductivity is one of the key parameters and determines the performance of a polymer electrolyte. The ionic conductivities of different weight percents of PPy incorporated esCPMs, that were derived by soaking esCPMs in an ionic liquid electrolyte, were determined by AC-impedance analysis shown in Fig. 4 and Table 1. When a small wt $\%$ of PPy nanoparticles was incorporated, it reduced the interaction between the polymer chains that decreased the degree of crystallinity of the polymer. The decrease in the degree of polymer crystallinity and the consequent increase of the amorphous content in the presence of PPy nanoparticles promoted the accumulation of electrolyte. Thus, the incorporation of $2 \mathrm{wt} \%$ of $\mathrm{PPy}$ incorporated esCPME improved the ionic conductivity with a maximum value of $26.10 \times 10^{-3} \mathrm{~S} \mathrm{~cm}^{-1}$ than the other $\mathrm{wt} \%$ of PPy incorporated esCPMEs. The ionic conductivity of esCPME(2wt\% PPy) was higher than esPME, but it was slightly lower than esCPME( $3 \mathrm{wt} \%$ PANI) $\left(31.44 \times 10^{-3} \mathrm{~S}\right.$ $\left.\mathrm{cm}^{-1}\right) \cdot{ }^{[24]}$ Beyond $2 \mathrm{wt} \%$, the addition of PPy blocked the polymer segmental movement, which returned to increase the ionic conductivity.

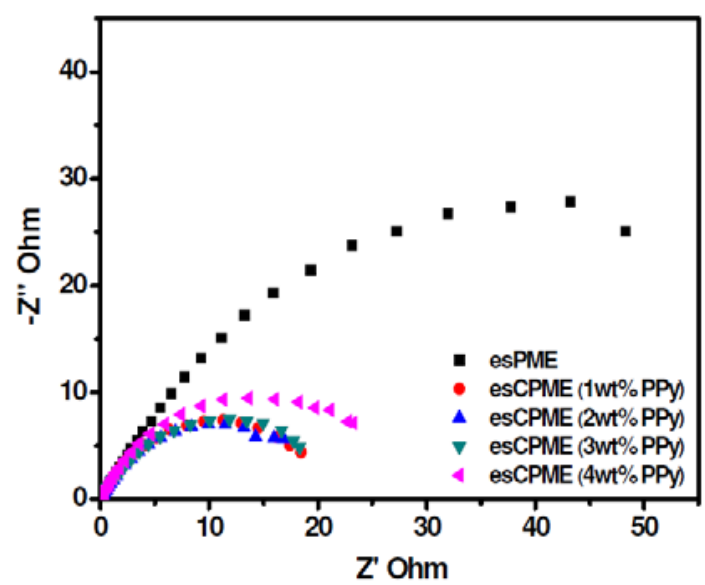

Fig. 4 Nyquist plots of different wt $\%$ of PPy incorporated esCPMEs on ionic conductivity.

Table 1. Influence of various wt $\%$ of PPy incorporated esCPMEs on ionic conductivity.

\begin{tabular}{cccc}
\hline Electrolyte & $\begin{array}{c}\text { Ionic conductivity } \\
\left(\times \mathbf{1 0}^{-3} \mathbf{S ~}_{\left.\mathbf{~} \mathbf{m}^{-\mathbf{1}}\right)}\right.\end{array}$ & $\mathbf{U} \mathbf{( \% )}$ & $\mathbf{P ~ ( \% )}$ \\
\hline esPME & 5.83 & 340 & 84.0 \\
esCPME (1wt\% PPy) & 24.81 & 374 & 88.0 \\
esCPME (2wt\% PPy) & 26.10 & 383 & 90.5 \\
esCPME (3wt\% PPy) & 24.31 & 365 & 87.0 \\
esCPME (4wt\% PPy) & 22.27 & 360 & 85.0 \\
\hline
\end{tabular}

Fig. 5(a) shows the schematic illustration of the formation of esCPME. The PPy incorporated in the threedimensional polymer nanofibrous framework presumably helps to enhance the charge transport kinetics as well as electrocatalytic behavior towards triiodides. The PPy nanoparticles provide electrical conductivity channels that favor electron conduction and extend the electrocatalytic 
reaction site from the $\mathrm{Pt}$ counter electrode/electrolyte interface to the three-dimensional network of esCPMEs. The extension of reaction sites accelerates the recovery of the redox couple (Fig. 5b) and thereby accelerates the diffusion of the $\mathrm{I}^{-} / \mathrm{I}_{3}{ }^{-}$redox couple. However, beyond the optimum concentration of $2 \mathrm{wt} \%$ of PPy, it appears to have a blocking effect due to the aggregation of PPy nanoparticles which hinders the transportation of the charge carriers and decreases the ionic conductivity. Thus, the DSSC based on 2 $\mathrm{wt} \%$ of PPy incorporated esCPME will provide an improved photovoltaic performance.

(a)

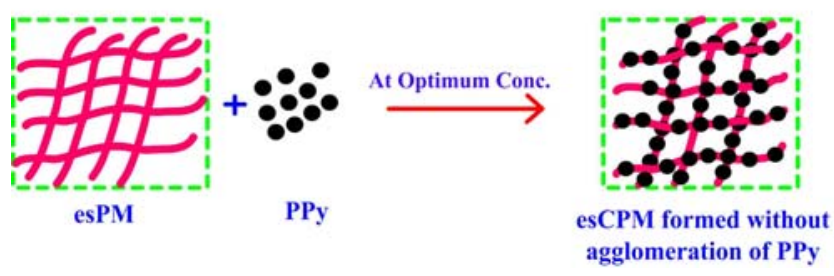

(b)

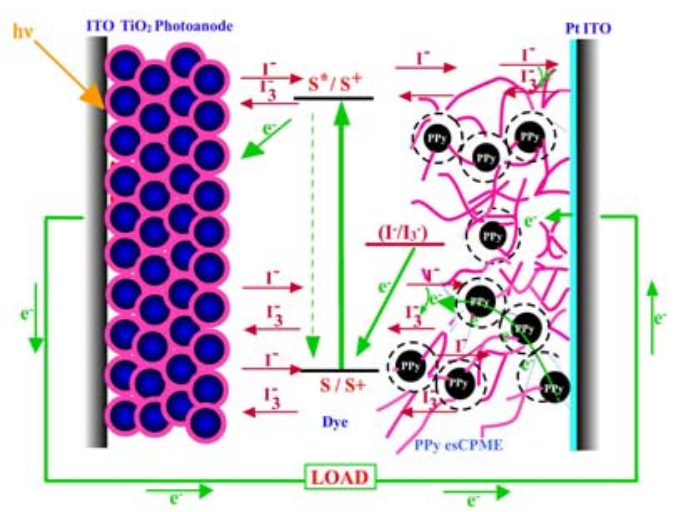

Fig. 5 (a) Schematic illustration on the formation of esCPME and (b) improvement in the ion transport mechanism.

\subsection{Tafel polarization studies}

To reconfirm the interfacial charge transfer (or) electrocatalytic activity of esCPMEs and counter electrode, Tafel polarization measurements were performed with the dummy cell containing esCPME sandwiched between the Pt- coated fluorine doped tin oxide (FTO) substrates [i.e. FTO/Pt/PME/ Pt/FTO]. Fig. 6 shows the Tafel polarization curves of esPME and esCPMEs. Typically, the Tafel polarization curve consists of three zones. The curve at the very high potential corresponds to the limiting diffusion zone, which is related to the transport of $\mathrm{I}_{3}{ }^{-} / \mathrm{I}^{-}$in the electrolyte. The middle potential range (with a sharp decrease) can be attributed to the Tafel zone where the current density of charge transfer appears. Finally, the curve at a low potential is attributed to the polarization zone. The polarization curve from the esCPME ( 2 wt.\% PPy) exhibits a large exchange current density $\left(J_{o}\right)$ in comparison to other electrolytes, suggesting that $2 \mathrm{wt} \%$ PPy incorporated esCPME showed a superior electrocatalytic activity for $\mathrm{I}_{3}^{-}$reduction. The PPy nanoparticles with a good electrical conductivity provide super highways for electron reflux from the counter electrode. The limiting current density $\left(J_{\text {lim }}\right)$ at very high potential depends on the diffusion of $\mathrm{I}_{3}{ }^{-} / \mathrm{I}^{-}$redox couple in the DSSC system that indicates a small $Z_{w}$. The $2 \mathrm{wt} \%$ PPy incorporated esCPME shows a high limiting diffusion current density when compared to other weight percentages. This behavior has a strong connection with the pore filling process, the high electrocatalytic activity of PPy presents in the electrospun polymer membrane electrolyte.

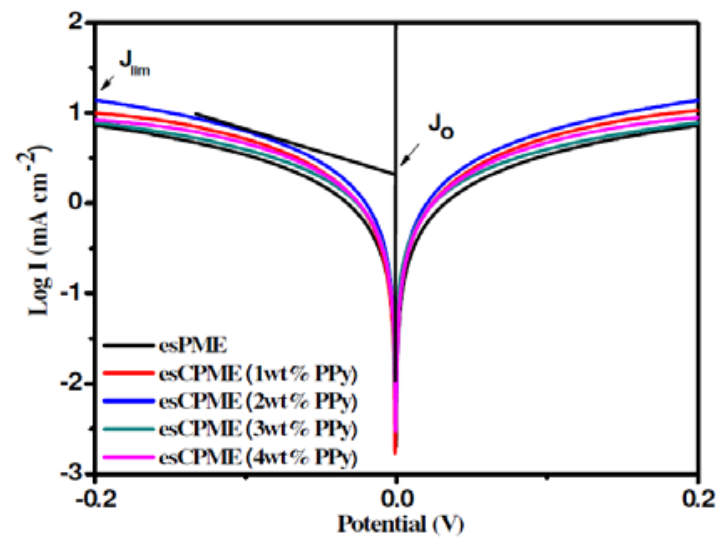

Fig. 6 Tafel polarization curves of the symmetrical cells assembled with various percentages of PPy incorporated esCPME.

\subsection{Photovoltaic performance studies}

The photocurrent density-voltage $(\mathrm{J}-\mathrm{V})$ curves obtained by the illumination with a light intensity of $100 \mathrm{~mW} \mathrm{~cm}$ (AM $1.5)$ for DSSC containing esCPME( $2 w t \%$ PPy) are shown in Fig. 7 (Table 2). In order to compare the photovoltaic performances, DSSCs based on esPME and esCPME are assembled and studied. The DSSC assembled using esCPME exhibits an enhanced photovoltaic performance compared to that assembled using esPME. This is due to the regular morphology of the nanofibers with increased porosity that facilitates the ion transport. The interfacial contact between the esCPME and dye-sensitized $\mathrm{TiO}_{2}$ photoanode also appears to improve the short circuit photocurrent density $\left(J_{S C}\right)$ value (Table 2$)$. The PPy nanoparticles in the esCPME enhanced the electrocatalytic activity of $\mathrm{I}_{3}{ }^{-} / \mathrm{I}^{-}$redox reaction, which leads to an increased $J_{S C}$ (from 13.10 to $13.90 \mathrm{~mA} \mathrm{~cm}{ }^{-2}$ ). Moreover, the higher electrocatalytic activity of esCPME reduces the overpotential, which in turn increases the $V_{o c}$. At the optimal concentration of PPy $(2 \mathrm{wt} \%)$, the reaction kinetics increases the photovoltaic performance. This may be due to the direct conductive pathway of electrons provided by PPy nanoparticles and their electrocatalytic activity. However, a further increase of PPy concentration leads to blocking of ionic mobility that decreases the photovoltaic performance. The DSSC assembled using $2 \mathrm{wt} \%$ PPy incorporated esCPME provides a PCE of $7.02 \%$, which is higher than that fabricated with esPME $(\eta=6.42 \%)$ and slightly lower than that fabricated with $3 \mathrm{wt} \%$ PANI incorporated esCPME $(\eta=7.20 \%)$. ${ }^{[17]}$ This might be due to the lower amorphocity $(29.87 \%)$ and 
higher ionic conductivity $\left(31.44 \times 10^{-3} \mathrm{~S} \mathrm{~cm}^{-1}\right)$ of PANI incorporated esCPME than PPy incorporated esCPME. ${ }^{[24]}$

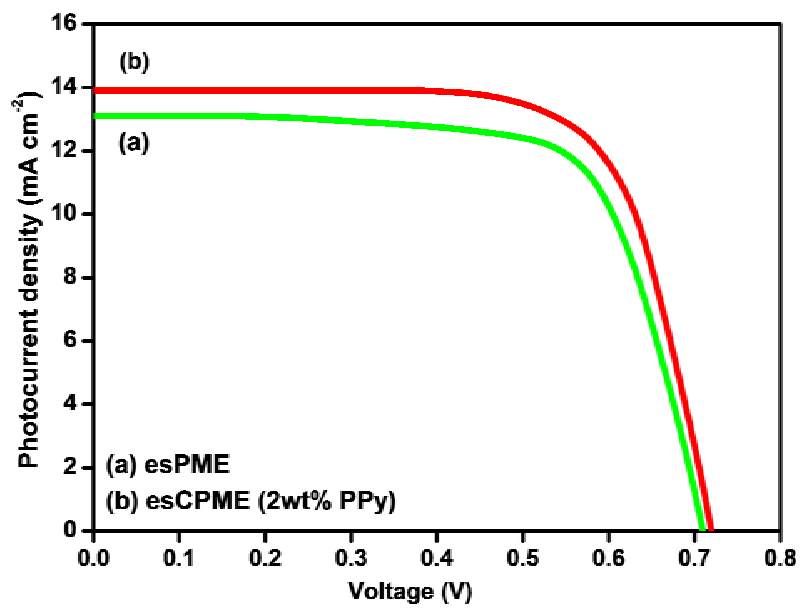

Fig. 7 Photocurrent density-voltage (J-V) curves of DSSC based on $2 \mathrm{wt} \%$ of PPy-esCPME and the conventional esPME.

Table 2. Photovoltaic performances of DSSCs based on $2 \mathrm{wt} \%$ PPy-esCPME and the conventional esPME.

\begin{tabular}{|c|c|c|c|c|}
\hline Electrolyte & $\begin{array}{c}\text { Jsc } \\
\left(\mathrm{mA} \mathbf{~ c m}^{-2}\right)\end{array}$ & $\begin{array}{l}\text { Voc } \\
\text { (V) }\end{array}$ & FF & $\begin{array}{c}\text { Efficiency } \\
(\%)\end{array}$ \\
\hline esPME & 13.10 & 0.71 & 69 & 6.42 \\
\hline esCPME (2wt $\%$ PPy) & 13.90 & 0.72 & 70 & 7.02 \\
\hline
\end{tabular}

\subsection{Stability studies}

Fig. 8 shows the long-term stability of DSSC revealing that those fabricated with the electrospun PVdF-HFP membrane are highly stable. The three-dimensional network of electrospun nanofibers provides sufficient mechanical strength and stability for both esPME and esCPME. Moreover, the high porosity and interconnectivity present in the esPM and esCPME (2wt.\% PPy) help to entrap a large quantity of liquid electrolyte and show no electrolyte leakage between the dye-adsorbed $\mathrm{TiO}_{2}$ electrode and $\mathrm{Pt}$ counter electrode, which attribute to the long durability of DSSC.

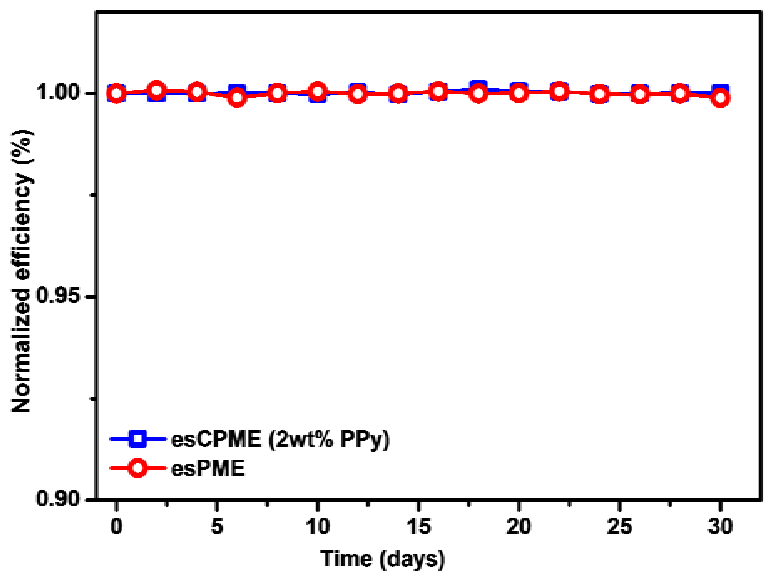

Fig. 8 Normalized light-to-electricity conversion efficiency of DSSC based on $2 \mathrm{wt} \%$ of PPy-esCPME and esPME.

\section{Conclusion}

The esCPM incorporated with PPy has a porous morphology formed by an interlayer of fibers and is capable of holding a large amount of the electrolyte. The incorporation of PPy into the esPM enhances its ionic conductivity and electrocatalytic activity. The performance of esCPME depends on the weight percentage of PPy nanoparticles employed. At an optimum concentration of $2 \mathrm{wt} \% \mathrm{PPy}$, the maximum solar to the electricity conversion efficiency of $7.02 \%$ was observed for DSSC assembled with the esCPME ( $2 \mathrm{wt} \%$ PPy) at an illumination intensity of $100 \mathrm{mWcm}^{-2}$.

\section{Acknowledgements}

One of the authors Dr. AS gratefully acknowledge the CSIR, New Delhi (Ref.No.01 (2810)14/EMR-II, dt. 26/04/2017) for the financial support and also the CIF of Pondicherry University for extending the instrumentation facilities.

\section{Supporting information}

Not applicable

\section{Conflict of interest}

There are no conflicts to declare.

\section{References}

[1] B. O'Regan and M. Gratzel, Nature 1991, 353, 737-740. doi: 10.1038/353737a0.

[2] C. P. Lee, C. T. Li and K. C. Ho, Mater. Today 2017, 20, 267-283. doi: 10.1016/j.mattod.2017.01.012.

[3] I. K. Ding, J. Melas-Kyriazi, N. L. Cevey-Ha, K. G. Chittibabu, S. M. Zakeeruddin, M. Grätzel and M. D. McGehee, Org. Electron. 2010, 11, 1217-1222. doi: 10.1016/j.orgel.2010.04.019.

[4] Y. Yu and M. Lira-Cantu, Phys. Procedia 2010, 8, 22-27. doi: 10.1016/j.phpro.2010.10.006.

[5] S. E. Hong, D. K. Kim, S. M. Jo, D. Y. Kim, B. D. Chin and D. W. Lee, Catal. Today 2007, 120, 413-419. doi: 10.1016/j.cattod. 2006.09.013.

[6] W. Zhang, Y. Cheng, X. Yin and B. Liu, Macromol. Chem. Phys. 2011, 212, 15-23. doi: 10.1002/macp.201000489.

[7] Y. F. Chan, C. C. Wang and C. Y. Chen, J. Mater. Chem. A 2013, 1, 5479-5486. doi: 10.1039/c3ta01684f.

[8] P. Y. Chen, C. P. Lee, R. Vittal and K. C. Ho, J. Power Sources 2010, 195, 3933-3938. doi: 10.1016/j.jpowsour.2009.12.086.

[9] Q. Li, Q. Tang, H. Chen, H. Xu, Y. Qin, B. He, Z. Liu, S. Jin and L. Chu, Mater. Chem. Phys. 2014, 144, 287-292. doi: 10.1016/j. matchemphys.2013.12.032.

[10] J. R. Kim, S. W. Choi, S. M. Jo, W. S. Lee and B. C. Kim, Electrochim. Acta 2004, 50, 69-75. doi: 10.1016/j.electacta.2004. 07.014.

[11] S. W. Choi, S. M. Jo, W. S. Lee and Y. R. Kim, Adv. Mater. 2003, 15, 2027-2032. doi: 10.1002/adma.200304617.

[12] S. W. Choi, J. R. Kim, Y. R. Ahn, S. M. Jo and E. J. Cairns, Chem. Mater. 2007, 19, 104-115. doi: 10.1021/cm060223+.

[13] M. Vinothkannan, A. R. Kim, K. S. Nahm and D. J. Yoo, RSC 
$A d v$. 2016, 6, 108851-108863. doi: 10.1039/c6ra22295a.

[14] M. Deka, A. K. Nath and A. Kumar, J. Membr. Sci. 2009, 327, 188-194. doi: 10.1016/j.memsci.2008.11.031.

[15] M. H. Jung, J. Power Sources 2014, 268, 557-564. doi: 10.1016/ j.jpowsour.2014.06.095.

[16] K. C. Huang, P. Y. Chen, R. Vittal and K. C. Ho, Sol. Energy Mater. Sol. C. 2011, 95, 1990-1995. doi: 10.1016/j.solmat.2010. 02.047 .

[17] Q. Li, X. Chen, Q. Tang, H. Xu, B. He and Y. Qin, J. Mater. Chem. A 2013, 1, 8055-8060. doi: 10.1039/c3ta11166k.

[18] S. Yuan, Q. Tang, B. He and P. Yang, J. Power Sources 2014, 254, 98-105. doi: 10.1016/j.jpowsour.2013.12.112.

[19] N. Senthilkumar, K. J. Babu, G. Gnana kumar, A. R. Kim and D. J. Yoo, Ind. Eng. Chem. Res. 2014, 53, 10347-10357.

[20] M. Ranjani, D. J. Yoo and G. Gnana kumar, J. Membr. Sci. 2018, 555, 497-506. doi: 10.1016/j.memsci.2018.03.049.

[21] S. Angaiah, V. Murugadoss, S. Arunachalam, P. Panneerselvam and S. Krishnan, Eng. Sci. 2018, 4, 44-51. doi: 10.30919/es8d756.

[22] A. K. Solarajan, V. Murugadoss and S. Angaiah, J. Appl. Polym. Sci. 2017, 134, 45177. doi: 10.1002/app.45177.

[23] A. K. Solarajan, V. Murugadoss and S. Angaiah, Sci. Rep. 2017, 7, 45390. doi: 10.1038/srep45390.

[24] V. Elayappan, V. Murugadoss, S. Angaiah, Z. Fei and P. Dyson, J. Appl. Polym. Sci. 2015, 132, 42777. doi: 10.1002/app.42777.
[25] E. Vijayakumar, A. Subramania, Z. Fei and P. J. Dyson, RSC Adv. 2015, 5, 52026-52032. doi: 10.1039/c5ra04944j.

[26] E. Vijayakumar, A. Subramania, Z. Fei and P. J. Dyson, J. Appl. Polym. Sci. 2015, 132, 42032. doi: 10.1002/app.42032.

[27] A. Subramania, N. T. Sundaram, A. R. Priya and V. G. Kumar, J. Membr. Sci. 2007, 294, 8-15. doi: 10.1016/j.memsci.2007.01. 025.

[28] N. Angulakshmi and M. A. Stephan, Electrochim. Acta 2014, 127, 167-172. doi: 10.1016/j.electacta.2014.01.162.

[29] A. R. S. Priya, A. Subramania, Y. S. Jung and K. J. Kim, Langmuir 2008, 24, 9816-9819. doi: 10.1021/1a801375s.

[30] G. G. Kumar, M. P. Balanay, R. Nirmala, D. H. Kim, T. R. Kumar, N. Senthilkumar, A. R. Kim and D. J. Yoo, J. Nanosci. Nanotechnol. 2016, 16, 581-587. doi: 10.1166/jnn.2016.10677.

[31] A. Subramania, E. Vijayakumar, N. Sivasankar, S. A. R. Priya and K. J. Kim, Ionics. 2013, 19, 1649-1653. doi: 10.1007/s11581013-0892-3.

[32] A. Solarajan, V. Murugadoss and S. Angaiah, Appl. Mater. Today 2016, 5, 33-40. doi: 10.1016/j.apmt.2016.09.002.

Publisher's Note: Engineered Science Publisher remains neutral with regard to jurisdictional claims in published maps and institutional affiliations. 